\title{
The maturation of cortical sleep rhythms and networks over early development
}

\section{Citation}

Chu, C.J., J. Leahy, J. Pathmanathan, M.A. Kramer, and S.S. Cash. 2014. “The Maturation of Cortical Sleep Rhythms and Networks over Early Development." Clinical Neurophysiology 125 (7) (July): 1360-1370. doi:10.1016/j.clinph.2013.11.028.

\section{Published Version}

doi:10.1016/j.clinph.2013.11.028

\section{Permanent link}

http://nrs.harvard.edu/urn-3:HUL.InstRepos:29663468

\section{Terms of Use}

This article was downloaded from Harvard University's DASH repository, and is made available under the terms and conditions applicable to Open Access Policy Articles, as set forth at http:// nrs.harvard.edu/urn-3:HUL.InstRepos:dash.current.terms-of-use\#OAP

\section{Share Your Story}

The Harvard community has made this article openly available.

Please share how this access benefits you. Submit a story.

\section{Accessibility}




\title{
The maturation of cortical sleep rhythms and networks over early development
}

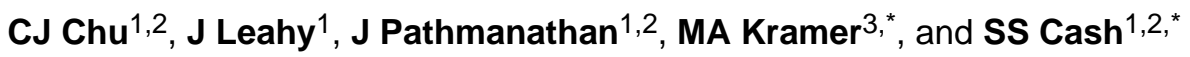 \\ ${ }^{1}$ Department of Neurology, Massachusetts General Hospital, Boston, MA 02144 \\ ${ }^{2}$ Harvard Medical School, Boston, MA 02144 \\ ${ }^{3}$ Department of Mathematics and Statistics, Boston University, Boston, MA, 02215
}

\begin{abstract}
Objective-Although neuronal activity drives all aspects of cortical development, how human brain rhythms spontaneously mature remains an active area of research. We sought to systematically evaluate the emergence of human brain rhythms and functional cortical networks over early development.
\end{abstract}

Methods-We examined cortical rhythms and coupling patterns from birth through adolescence in a large cohort of healthy children $(n=384)$ using scalp electroencephalogram (EEG) in the sleep state.

Results-We found that the emergence of brain rhythms follows a stereotyped sequence over early development. In general, higher frequencies increase in prominence with striking regional specificity throughout development. The coordination of these rhythmic activities across brain regions follows a general pattern of maturation in which broadly distributed networks of lowfrequency oscillations increase in density while networks of high frequency oscillations become sparser and more highly clustered.

Conclusion-Our results indicate that a predictable program directs the development of key rhythmic components and physiological brain networks over early development.

Significance-This work expands our knowledge of normal cortical development. The stereotyped neurophysiological processes observed at the level of rhythms and networks may provide a scaffolding to support critical periods of cognitive growth. Furthermore, these conserved patterns could provide a sensitive biomarker for cortical health across development.

\section{Keywords}

Development; brain rhythms; brain networks; functional connectivity; sleep; EEG

(c) 2013 International Federation of Clinical Neurophysiology. Published by Elsevier Ireland Ltd. All rights reserved.

Corresponding Author: Catherine J. Chu, MD, 175 Cambridge Street, Suite 340, Massachusetts General Hospital, Boston, MA 02114, USA, Tel: +1-617-726-6540, Fax: +1-617-726-0230, cjchu@ hms.harvard.edu.

*Professor Kramer and Dr. Cash contributed equally to this work as senior authors

Publisher's Disclaimer: This is a PDF file of an unedited manuscript that has been accepted for publication. As a service to our customers we are providing this early version of the manuscript. The manuscript will undergo copyediting, typesetting, and review of the resulting proof before it is published in its final citable form. Please note that during the production process errors may be discovered which could affect the content, and all legal disclaimers that apply to the journal pertain. 


\section{INTRODUCTION}

Over the course of post-natal development, the human brain transforms from a relatively quiescent structure into a highly complex organ capable of increasingly sophisticated cognitive processes. Throughout these critical periods, neuronal activity guides all aspects of cortical development from neurogenesis, neuronal differentiation and migration, to synaptic maturation and pruning (Komuro et al., 1993; Katz and Shatz, 1996; Kriegstein, 2005). A substantial literature supports the hypothesis that neuronal oscillations serve to orchestrate complex neuronal assemblies through transiently coupled physiological rhythms across multiple temporal and spatial scales (reviewed in Buszaki 2004). Such coordinated cortical functional networks underlie multiple cognitive processes (Gray et al., 1989; O'Keefe et al., 1993; Fries 2005; Buszaki and Draguhn, 2004) and have been found to be disrupted in many cortical diseases (e.g. Uhlhaas and Singer, 2010; Sun et al., 2012, Kramer and Cash, 2012). How human brain rhythms and circuits emerge and mature during critical periods of cortical development remains an active area of research. The sleep electroencephalogram (EEG) provides a unique in vivo opportunity to observe spontaneous cortical voltage activity across interacting brain regions over the course of post-natal cortical development, providing a window into the intrinsic maturation of brain rhythms across brain regions. Signal processing techniques tailored for neurophysiological data enable principled evaluation of the emergence of these neuronal rhythms and the large-scale cortical ensembles (functional networks) they coordinate.

Prior work evaluating developing cortical rhythms and connectivity patterns during sleep suggests rich dynamics, but remains incomplete due to sparse electrode sampling, small number of subjects, and limited evaluation of pediatric age ranges (Kuks et al., 1988; Sterman et al., 1977; Gadreau et al 2001; Jenni and Carskadon 2004; Jenni et al., 2005; Campbell and Feinberg, 2009; Myers et al 2010; Kurth et al, 2010; Tarokh et al., 2010; Feinberg et al., 2011). We examined cortical rhythms and brain connectivity patterns from birth through adolescence in a large cohort of developmentally normal children using scalp EEG in the sleep state. We found that brain rhythms and connectivity patterns change dramatically over childhood, but follow a remarkably stereotyped sequence. In general, higher frequencies increase in prominence with age while there is striking regional specificity throughout development. The maturation of coupling patterns follows a general pattern in which low-frequency networks increase in density but are broadly distributed across childhood and adolescence and high frequency networks become sparser but highly clustered across development. Our results indicate that a predictable program directs the development of key rhythmic components and physiological brain networks over early development. This work provides a foundation upon which to better understand the neurophysiological scaffolding that supports normal brain development and ultimately, how alterations in these precisely timed sequences may relate to and even anticipate disease. 


\section{MATERIALS AND METHODS}

\section{Subjects and EEG recordings}

Subjects age 0-18 years with normal EEG recordings (as defined by clinical electroencephalographers independent from this study) were retrospectively identified from recordings performed at Massachusetts General Hospital between 2/1/2002 and 5/1/2012 $(n=4175)$. Clinical chart review was performed and only those children with documented normal neurodevelopment and non-epileptic events were included for analysis.

Neurodevelopmental status was determined from chart review of the clinical assessments just prior to or following the EEG recording. Patients that received alcohol, sedatives, anticonvulsant medications, or neuro-active medications during the recording period were excluded. Children born prematurely ( $<38$ week gestational age) were excluded. Subjects in whom the EEG recordings had excessive muscle artifact were also excluded (n=19). 384 subjects (187 females, 197 males, aged 1 day through 18 years 11 months) met inclusion criteria. Identified non-epileptic events leading to diagnostic evaluation in these subjects are listed in Table 1. Subjects were placed into age groups according to age at time of EEG with groups defined by month from 0-23 months, by 6 months interval for ages $24-59$ months, and by 12 month intervals from 60-216 months. This grouping maintained approximate group sizes across ages ( $\mathrm{n}=8.8 \pm 3.4$ per age bin) and allowed rapid changes in cortical voltage properties over infancy to be captured.

To increase the total number of subjects in this analysis, we included children with a history of provoked seizure (e.g., febrile seizure, post-concussive seizure, and post-syncopal seizure) as these events do not indicate epilepsy and these subjects have a low risk of subsequent epilepsy or neurological disease (Hesdorffer et al., 1998; Shinnar and Glauser, 2002). On subgroup analysis, we found no difference in mean spectral power between those with a history of seizures and those without in any of the frequency bands evaluated ( $p>0.7)$ and no difference in functional network density (defined below, $\mathrm{p}>0.99$ ) between subjects without a history of seizure $(n=296)$ and those with a history of provoked seizure $(n=88)$.

Recordings included electrooculogram (two channels), EEG (19 Ag/AgCl electrodes placed according to the 10-20 international system: FP2, F4, C4, P4, O 2, F8, T4, T6, Fz, Cz, Pz, Fp1, F3, C3, P3, O1, F7, T3, and T5) and electrocardiogram using a standard clinical recording system (Xltek, a subsidiary of Natus Medical). Signals were sampled at 200, 256, 500 or $512 \mathrm{~Hz}$ and stored on a local server. Analysis of the data from these subjects was performed retrospectively under protocols approved and monitored by the local Institutional Review Board according to National Institutes of Health guidelines.

\section{EEG Pre-processing}

EEG recordings were manually reviewed by an experienced electroencephalographer (C.J.C. and/or J.P.) and large movement and muscle artifact removed. EMG spectra is known to have broadband activity $>25 \mathrm{~Hz}$ and is maximal in the higher frequencies $(40-90 \mathrm{~Hz})$ temporally (Gasser et al, 2005). As a conservative measure to minimize the impact of low amplitude muscle artifact not identified by visual analysis contaminating analysis of high frequencies $(>25 \mathrm{~Hz}$ ), we identified outliers that did not have the expected steep fall-off of 
power with frequency (Freeman et al., 2000). To do so, we computed a linear fit of the log power versus the log frequency for frequencies between $30-50 \mathrm{~Hz}$ and $65-95 \mathrm{~Hz}$ in temporal leads (selected due to sensitivity for temporal muscle artifact) for each subject. If the slope of this fit exceeded -1 , indicative of a slow decrease in power with frequency (Partha and Hemant, 2008), we excluded these subjects from analysis.

Stage 2 (N2) sleep was identified by visual analysis as per standard criteria (Silber et al., 2007). N2 sleep was selected for analysis for several reasons. First, N2 sleep provides a state of relative uniformity amongst subjects, minimizing the confounds of attention and environmental-specific stimuli, thereby emphasizing spontaneous rhythms intrinsic to the developing system. Second, N2 sleep is contaminated by minimal muscle and movement artifacts, providing a greater signal to noise ratio (Scher, 2008; Eeg-Olofson, 1980). Third, $\mathrm{N} 2$ sleep is the most reliably identified sleep state among experienced neurophysiologists (Rosenberg et al, 2013). Finally, we have previously shown that there is the least within individual variation in EEG functional networks in the N2 sleep state, allowing more sensitive identification of population trends (Chu et al, 2012).

For neonates without clear sleep spindles, we selected for quiet sleep according to standard criteria (Anders, 1974). After referencing, the mean voltage was subtracted from each voltage tracing. The $\mathrm{N} 2$ sleep data were concatenated to generate a single continuous file of 100s duration. We have previously demonstrated that this epoch size is sufficient to produce high similarity between networks inferred from N2 sleep samples (see Chu et al., 2012).

\section{Volume conduction}

Spatial blurring of the voltage signal propagating from the cortex to the scalp is a known limitation of EEG recordings. Here, we have implemented several measures to reduce the impact of volume conduction on the scalp voltage recordings prior to analysis. First, we have utilized a nearest-neighbor Laplacian montage to maximize identification of local potential deviations (Nunez and Srinivasan, 2006). This referencing technique minimizes the impact of volume conducted signals in both anterior-posterior or midline-lateral dimensions and performed best in our simulations (data not shown) comparing physical reference, bipolar reference, and common average reference procedures on a three spherical head model (see Nunez and Srinivasan, 2006). In spite of careful referencing, random coupling is known to occur between multi-electrode recordings. In order to ascribe confidence to the statistical associations measured, we employed an analytical technique for both the cross correlation and the coherence measures to assess the significance of coupling relative to the background activity (see below, Kramer 2009). Finally, to further reduce the potential impact of volume conduction on the voltage recordings, we ignored data in which maximal coupling was identified at zero lag or zero phase. A detailed discussion of the robustness of this method can be found in Chu et al., 2012.

\section{Power spectra}

We computed the power spectra using the multitaper method (time bandwidth product of 3 , 3 second windows, 5 tapers, $1 \mathrm{~Hz}$ frequency resolution). We chose this window size to balance signal stationarity and frequency resolution. In order to reduce variability in spectra 
measurements due to trivial contributions from changes in head size and skull thickness across development (Law, 1993; Bronzino, 1999), we report the relative changes in power in each frequency band after normalizing to the total power. To illustrate that the spectral analysis technique employed here is robust to changes in head size and skull thickness, we performed the following simulation study. We considered a 3-shell (scalp, skull, cortex) spherical head model (Nunez and Srinivasan, 2006) in two configurations: 1) An "Adult" model with adult geometries $(9.2 \mathrm{~cm}$ scalp radius, $6.5 \mathrm{~mm}$ skull thickness, $8.25 \mathrm{~cm}$ cortex radius). 2) An "Infant" model with infant geometries ( $7.0 \mathrm{~cm}$ scalp radius, $4 \mathrm{~mm}$ skull thickness, $6.3 \mathrm{~cm}$ cortex radius). We assume in both models the same standard values of resistivities (Nunez and Srinivasan, 2006). For each model, we simulated voltage activity using $~ 1100$ dipole sources evenly distributed just below the cortical surface (Nunez and Srinivasan, 2006). We then observed the simulated scalp EEG data from each model configuration using sensors placed following the standard scalp EEG cap (Fig. 1A). We note that although the presence of sutures and the density of the bone are all expected to impact the resistance of the skull to cortical activity, in empirical studies, skull resistance is found to be linearly correlated to skull thickness (Law, 1993).

We first simulated each cortical dipole source (there are 1100 ) as pink noise. We note that pink noise captures an important qualitative feature of observed brain activity: the reduction in signal power with frequency. Both the "Adult" and "Infant" model configurations possess the same number of sources and identical source activity; the only difference between the two configurations is the head geometry. We analyze the simulated EEG data using the same procedures applied to the clinical EEG data described in the manuscript; namely, we apply the Laplacian reference, bandpass filter the data $(1-50 \mathrm{~Hz})$, and subtract the mean from each electrode derivation. We then computed the power spectrum of these data for each 2 second window, normalized by dividing by the total power for each electrode, as we did for the clinical EEG data described in the manuscript. We show the ratio of the power spectra from the Adult model configuration to the Infant model configurations in Fig. 1B (blue curve). These results indicate that the analysis approach mitigates the impact of volume conduction on the spectral results. We note that repeating the analysis without normalization of the data results in lower power at all frequencies studied $(1-50 \mathrm{~Hz})$ in the Adult model configuration (red curve in Fig. 1B), as expected due to the spatial blurring of the thicker skull.

The power per $1 \mathrm{~Hz}$ frequency was computed for each window for each electrode and scaled by the total power. The average scaled power for each frequency and for each frequency band of interest (delta $(1-4 \mathrm{~Hz})$, theta $(4-8 \mathrm{~Hz})$, alpha $(8-12 \mathrm{~Hz})$, beta $(12-20 \mathrm{~Hz})$, high beta $(20-30 \mathrm{~Hz})$, low gamma $(30-50 \mathrm{~Hz})$, high gamma $(65-95 \mathrm{~Hz})$ were computed for each subject, averaged and reported as a mean with standard error of the mean for each age group using Chronux (Mitra and Bokil, 2008) and custom software developed in MATLAB.

\section{Functional network construction}

To assess the associations between voltage activities recorded at two electrodes, we use two measures of linear coupling: cross correlation and coherence. Many linear and nonlinear measures can be used to assess signal coupling. We use the cross correlation and coherence measures because analytic and computationally efficient tests for inference of significant 
coupling exist. To correct for multiple comparisons, a linear step-up false detection rate controlling procedure was used with $\mathrm{q}=0.05$. For this choice of $\mathrm{q}, 5 \%$ of the edges are expected to be falsely declared (Benjamini and Hochberg, 1995). A detailed discussion of the statistical testing applied to one of these measures can be found in Kramer et al, 2009.

Functional networks were constructed using both broad- and narrowband analysis. To construct the functional networks, the prepared EEG data were divided into discrete $2 \mathrm{~s}$ windows. For broadband analysis, the data are filtered with high- and low-pass filters (thirdorder Butterworth, zero-phase shift digital filtering) for frequencies of interest (1-50 Hz). Windows that contained concatenated data from noncontiguous time points are discarded. Within each window, the data are normalized from each electrode to have zero mean and unit variance before coupling analysis. We then calculate the maximal cross correlation between all electrode pairs, allowing a lag of $\pm 500 \mathrm{~ms}$. The choice of lag time was selected to provide sufficient variance for significance testing (Kramer et al., 2009). For analysis of narrower frequency bands of interest, we applied the coherence measure on unfiltered data. For this, we used a multitaper method and compute the coherence between signals at center frequencies $(2.5,6,10,16,25,40$, and $80 \mathrm{~Hz})$ for all electrode pairs. We set our parameters to allow for accurate resolution of classically recognized EEG band frequencies observed in the scalp EEG (Buzsaki, 2004; Darvas et al., 2010; Piantoni et al., 2013) and peak signals identified on power spectral analysis (1-4 Hz: time bandwidth product (NW) 2.5, 4 tapers; 4-8 Hz: NW 4, 7 tapers; 8-12 Hz NW 4, 7 tapers; 12-20 Hz: NW 8, 15 tapers; 20-30 Hz: NW 10, 19 tapers; 30-50 Hz: NW 10, 19 tapers; 65-95 Hz: NW 30, 59 tapers). For each $2 \mathrm{~s}$ window, the connectivity of the EEG data is represented as a network in the form of an undirected binary adjacency matrix M. Significant coupling (either cross correlation or coherence) between two electrodes $\mathrm{i}$ and $\mathrm{j}$, indicating an edge in the functional network, is represented as $\mathrm{M}(\mathrm{i}, \mathrm{j})=\mathrm{M}(\mathrm{j}, \mathrm{i})=1$. If electrodes $\mathrm{i}$ and $\mathrm{j}$ lack significant coupling, $\mathrm{M}(\mathrm{i}, \mathrm{j})=$ $\mathrm{M}(\mathrm{j}, \mathrm{i})=0$. Diagonal matrix elements, $\mathrm{M}(\mathrm{i}, \mathrm{i})$, are always set to 0 . The binary networks generated from each window are averaged across time to create weighted functional networks representative of fifty, $2 \mathrm{~s}$ epochs for each subject. Weighted networks are then averaged for each age group.

We note that the changing skull geometry across developmentis expected to have a great impact on surface EEG coupling measures because of distortions in the spatial extent of volume conduction on the cortical signals. Here we employ several measures to conservatively reduce the impact of volume conduction (see Section Volume Conduction). To illustrate that the network inference procedure employed here is robust to changes in head size and skull thickness, we performed the following simulation study. We considered the same 3-shell (scalp, skull, cortex) spherical "Adult" and "Infant" head models described above (see Section Power Spectra and Fig. 1A). We then simulated the dipole source activity as pink noise, and constructed functional networks from the simulated scalp EEG data. To do so, we employed the same network inference procedure described in the manuscript. We focus here on the cross correlation measure. We find no difference in the number of edges detected in the two model configurations (Fig. 1C): in 1000 simulations of the model, $98 \%$ of the networks in both model configuration possessed 0 or 1 edge, and $99.5 \%$ of networks possessed 2 or fewer edges. For this simulation scenario, all of the edges are spurious (because the dipole sources lack any organization - they're all uncorrelated 
pink noise activity). Consistent with this scenario, we tend to find networks with very few edges. We note that, in the clinical EEG data, we find densities in the $120 \mathrm{~m}$ and older consistent with 5 or more edges, well above the number of spurious edges detected here.

We also constructed functional networks for the simulated data with correlated source activity. To do so, we simulated two cortical dipole sources with five times larger magnitude than the other (uncorrelated, pink noise) sources. These two cortical dipole sources possessed correlated dynamics: for a $2 \mathrm{~s}$ interval of data, the sources exhibited $15 \mathrm{~Hz}$ oscillations for the middle $1 \mathrm{~s}$ interval of data, with a delay of $100 \mathrm{~ms}$ between the two sources. We simulated the model as above, and inferred functional networks from the simulated EEG scalp activity using the cross correlation. We find that the functional network procedure successfully detects an increase in the number of edges; compare the left (uncorrelated) and right (correlated) panels of Fig. 1C. In this way, the inferred network from the simulated scalp EEG reflects increased coupling between cortical sources. Although this simulation study necessarily simplifies aspects of the true human head (e.g., a spherical shell model), these results provide additional confidence that the analysis procedures are robust to changes in head size, skull thickness, and electrode distance.

\section{Network measures}

For characterization of global network connectivity, we computed the average density and global clustering coefficient of the networks (Rubinov and Sporns, 2010; Newman, 2010). The average density of a network, $d$, is defined as the number of edges observed divided by the total number of possible edges. The average density was computed for each subject and the mean per age group computed. The global clustering coefficient, $C$, is defined as the average of local clustering coefficients of all nodes, where local clustering coefficient is defined as the proportion of edges between the neighbors of a node divided by the total number of possible edges between the neighbors of the node. To compute these network measures, we used algorithms from the Brain Connectivity Toolbox (Rubinov and Sporns, 2010). Disconnected nodes were counted as contributing zero triangles in the calculation of global clustering coefficient. We note that clustering coefficient measures are correlated with density (Faust, 2007). Thus, for each network, we normalize the computed clustering coefficient against the average clustering coefficient generated from 500 randomized models in which the density is preserved (Newman, 2010). Although many other measures (weight dispersion, assortativity, global efficiency, and path length) were considered to further characterize the networks, the sparsity of nodes ( $n=19$ electrodes) and very low densities identified for each network limited the utility of additional measures.

\section{Statistical Tests}

Differences in group means were identified using a two-way ANOVA test with variables age and statistic of interest (mean power and density) and a Tukey least square difference test to correct for multiple comparisons. Subgroup analyses were performed with variables a) spectral power or density and b) subgroup (males versus females and history of provoked seizure versus no history of seizure) using a 2-tailed t-test. Corrections for multiple comparisons were performed using the Bonferroni method. 


\section{RESULTS}

\section{Cortical rhythms are age specific}

Visual inspection of power spectra revealed stereotyped frequency specific patterns across development (Fig. 2A). Lower frequencies dominate in all ages, with greatest prominence in infancy. Higher frequencies increase in power over childhood with prominent spectral peaks in the low beta range $(12-20 \mathrm{~Hz})$ appearing during infancy, and broad increases in the high beta and low gamma range $(20-40 \mathrm{~Hz})$ persisting from 6 months through 3 years of age, at which point beta activity $(12-20 \mathrm{~Hz})$ becomes prominent. A discrete peak in alpha power $(11 \mathrm{~Hz})$ is evident from 16 months. A marked increase in power in the high gamma range (50-100 Hz) is observed in adolescence (Fig. 2A).

In order to evaluate further the relationship between the frequency of cortical rhythms and age, we computed the mean power across seven frequency bands: delta $(1-4 \mathrm{~Hz})$, theta $(4-8$ $\mathrm{Hz})$, alpha $(8-12 \mathrm{~Hz})$, low beta $(12-20 \mathrm{~Hz})$, high beta $(20-30 \mathrm{~Hz})$, low gamma $(30-50 \mathrm{~Hz})$, and high gamma $(55-95 \mathrm{~Hz})$. Consistent with prior reports, we found no difference in spectral power between males and females in any age bin (t-test, p>0.27; Jenni et al., 2005; Baker et al, 2011).

There was a significant relationship between spectral power and age in each of the frequency bands (ANOVA, $\mathrm{p}<0.0001$; Fig. 1B). In the delta band, power initially increases in early infancy followed by a dramatic decrease from age 6 months to approximately 15 months followed by a plateau through age 10 years, prior to a second decline observed in adolescence. In the theta, alpha, and low beta bands, power increases consistently with age. In the high beta and low gamma bands, power generally increased with age through 18 months with a relative plateau thereafter. Power in the high gamma band demonstrates a Ushaped trend with an initial drop over the first 6 months of life followed by a steady increase through adolescence (Fig. 2B). Our findings are in concert with the spectral features previously reported in neonates (Myers et al., 2010; Sankupellay et al., 2011), children (Gaudreau et al., 2001; Smit et al., 2012) and adolescents (Gaudreau et al., 2001; Feinberg et al., 2011; Smit et al., 2012). Here we show that across ages, there is a gradual reduction in power for low frequencies, and gradual increase in power for higher frequency bands across development, interspersed with additional structure in the first months of life.

\section{Cortical rhythms have regional specificity over development}

In order to evaluate the evolution of rhythms in different cortical regions across development, we computed the power spectra of the EEG activity at each electrode for each age group. We computed the mean power across each of the 7 frequency bands and found a significant relationship between age and frequency band for each cortical region evaluated (ANOVA, $\mathrm{p}<0.0001$ for all tests). Visual inspection revealed several spatially specific frequency patterns across development (Fig. 2). In particular, theta and alpha activity increase with age primarily in the posterior regions (Fig. 3A, purple arrows). Prominent bursts of activity are present in the midline and frontocentral regions at $14 \mathrm{~Hz}$ at age $2 \mathrm{~m}$ (Fig. 3A, broad white arrows) and later at $\sim 6 \mathrm{~m}$ in the left temporal regions (Fig. 2, pink arrow). A streak of narrow alpha activity appears, centered at $11 \mathrm{~Hz}$ in midline, frontal and 
temporal regions by age $18 \mathrm{~m}$ (Fig. 3A, narrow white arrows). In the high beta and low gamma frequencies, prominent bursts of activity are broadly present from $6 \mathrm{~m}-5 \mathrm{y}$, but most prominent in the central and temporal regions (Fig. 3A, gray circles). In these same regions, though most prominent centrally, high frequency power $(>65 \mathrm{~Hz})$ increases from childhood into teenage years (Fig. 3A, black triangles).

In order to further evaluate the relationship between the topography of cortical rhythms across development, we computed the mean normalized power across seven frequency bands: delta (1-4 Hz), theta (4-8 Hz), alpha $(8-12 \mathrm{~Hz})$, low beta $(12-20 \mathrm{~Hz})$, high beta (20$30 \mathrm{~Hz})$, low gamma $(30-50 \mathrm{~Hz})$, and high gamma $(55-95 \mathrm{~Hz})$ at each electrode at each age. There is a significant relationship between spectral power and age in each of the frequency bands in each electrode (ANOVA, p<0.0001; Fig. 3B). In the delta band, the dramatic rise and fall in power in the first 15 months followed by a second fall in adolescents is evident in all electrode locations, though most prominent in the temporal and occipital electrodes. In the remaining frequency bands evaluated, marked spatial structure was present with age (Fig. 3B).

To summarize, lower frequency theta and alpha $(<10 \mathrm{~Hz})$ increase in prominence in the posterior regions over childhood. Higher frequencies demonstrate prominent activity in the frontal central and midline in early infancy $(10-15 \mathrm{~Hz})$ and centrotemporal regions during toddler years and childhood $(20-50 \mathrm{~Hz})$ and adolescence $(20-95 \mathrm{~Hz})$.

\section{Frequency specific cortical networks emerge across development}

In order to evaluate for frequency-specific coupling patterns between different brain regions, we computed the mean functional networks in each frequency bands for each age group. Visual inspection of the average networks across development revealed striking topological organization (Fig. 4). In general, functional network patterns between cortical regions are grossly symmetric between hemispheres across development. The earliest patterns of cortical brain connectivity appear as diffuse connections in the low beta frequencies in early infancy that are most prominent in midline regions, followed by prominent connectivity in bilateral anterior regions in the theta frequencies in the second year of life. Prominence of anterior and lateral to midline connections in delta and alpha frequencies develop over toddler years and childhood. High beta and low gamma frequency networks highlight neighboring anterior, lateral and posterior brain regions at all ages. High gamma coupling is seen diffusely and non-specifically initially and later coupling is most prominent between adjacent brain regions by late childhood and teenage years.

In summary, frequency specific coupling patterns are observed at each age. In low frequency bands, large-scale patterns covering broader spatial regions are noted to emerge in later childhood. In higher frequency bands, the opposite pattern is observed, with increased local connections observed with age. These results are consistent with observations that low frequency networks cover broader spatial regions and may be reflective of underlying structure, while higher frequency networks may play an essential role in integrating focal regions in transient cognitive tasks (Singer, 1999; He and Raichle, 2009; Baria et al., 2011). 


\section{Functional network structure evolves across normal development}

In order to characterize the strength and organization of coupling between brain regions at different ages, we computed the average network density and clustering coefficient for each age group in each frequency band. Network density provides an overall measure of network connectedness. This measure is bounded between 0 and 1 with higher numbers reflecting networks with more edges (i.e., denser networks). We found a prominent relationship between network density and age in the broadband networks, where network density steadily increased over the course of development (ANOVA, $\mathrm{p}<0.00001$ ) with a dramatic rise in density seen after age 5 years (see Fig. 5). We found no difference in density between males and females in any age group (t-test, $\mathrm{p}>0.99$ ). The findings in the broadband networks were reflected in the delta and theta band networks; both low frequency networks exhibited initial low densities during infancy which increased prominently after age 5 years (ANOVA, $\mathrm{p}<0.00001)$. In the alpha frequency networks, high variability between subjects was present at most ages, though some age-specific structure was present $(\mathrm{p}=0.003)$. In the low beta and high beta networks, initial peaks in density were present in early infancy followed by a relative plateau through adolescence (ANOVA, $\mathrm{p}<0.00001$ ). A similar trend was present in the low gamma networks (ANOVA, $\mathrm{p}=0.004$ ).

Global clustering coefficient measures the tendency of nodes in a network to cluster together, here reflecting the tendency of groups of brain regions to coordinate beyond random. Typically this measure is bounded between 0 and 1 ; however, the clustering coefficient is highly coupled to network density, so we have normalized our measure such that values $>1$ reflect a tendency to cluster beyond that expected in random networks (see Methods). For most frequency bands, the normalized clustering coefficient was found to be near-random with no relationship with age (delta, alpha, low beta, high beta, low gamma, ANOVA, $\mathrm{p}>0.05$ ). In broadband, theta, and high gamma networks, we found a significant relationship between clustering coefficient and age (Fig. 5, right; ANOVA, $\mathrm{p}<0.002$ for each frequency band shown). In broadband networks, clustering generally decreased with age. In theta band networks, peaks in clustering were evident during early infancy. In high gamma networks, clustering coefficient increased with age. Other variations in clustering coefficient limited to a single age bin were also present, but were felt to be likely due to artifact given the small sample sizes within each age group.

In summary, we found a general increase in integration between brain regions over the course of development as measured by increased network density with age in the broadband and low frequency networks. More complex structure is evident in mid-range frequencies with alternating periods of heightened and reduced integration observed across development. In high gamma frequency networks, density decreases with age. In low and high frequency bands, as network density decreases, global clustering coefficient increases, suggesting that clusters of brain regions are preferentially integrated in sparse networks; in particular, sparser, more clustered high frequency networks are present with age.

\section{DISCUSSION}

Cortical rhythms are presumed to be an essential part of brain function. The maturation and interregional coupling of these rhythms has long been recognized as precisely timed, 
although enigmatic process (Eeg-Olofson, 1971; Scher and Loparo, 2009). Here, we have evaluated rhythms and cortical functional networks in a large population of infants and children across development in the sleep state. We have found that cortical rhythms follow an orchestrated maturational sequence across development and that these sequences are nonlinear and regionally specific. In general, we have found that after mid-infancy, the relative contribution of power in low frequencies decreases, and that of higher frequencies increase with age. More specifically, we observed markedly stereotyped sequences with bursts of increased power seen in each cortical region at different ages and unique frequency bands. We also demonstrated dominant network connectivity patterns integrating distant brain regions over the course of development with distinct characteristics in each frequency band. Our findings are consistent with recent work over smaller and more sparsely sampled age ranges (Smit et al., 2012; Myers et al., 2012; Feinberg et al., 2011), and helps to uncover the neurophysiological signatures of the scheduled windows of cortical maturation and integration that occurs across development.

Much prior work has been done to evaluate spectral features in EEG recordings over development. In spite of some methodological differences in EEG analysis techniques, we find consistent results, though here complemented by greater age and spatial resolution. In a longitudinal study, Sankupellay and colleagues evaluated 34 healthy infants power spectra at the C3 electrode at 2 weeks, 3, 6, 12, and 24 months and reported peaks in power at $\sim 13 \mathrm{~Hz}$ at 3,6, and 12 months (Sankupellay et al, 2011). We find that this peak is evident earlier, at 2 months $(\sim 14 \mathrm{~Hz})$, and maximal in the midline and frontocentral regions. Sankupellay and colleagues also report a peak at $\sim 11 \mathrm{~Hz}$ at 24 months. Here, we find this peak is evident by 18 months and maximal in the midline, frontal and temporal regions. Prior work in adolescents using longitudinal datasets has reported a precipitous decline in NREM delta power starting at age 11 years maximal in occipital regions (Campbell and Feinberg, 2009; Baker et al, 2011). We expand this work across development and show that occipital delta power initially increases in early infancy followed by a dramatic decrease from 6-15 months followed by a relative plateau through age 4 years, which is then followed by the decline reported in adolescence. Furthermore, we find a marked increase in delta power from ages 5-8 years, most prominent in the temporal regions. Kurth et al evaluated cortical rhythms using high density EEG in 41 subjects from ages 2.4-19.4 years. These authors report that the maximal delta activity shifts from posterior to anterior regions between school age (5-8 yr) and early adolescents (11-14 yr). We did not find this topographical shift in delta power in our subjects, likely due to differences in EEG analysis techniques. In order to minimize the impact of skull size and bone resistivity on power measures between individuals and electrode locations (Law, 1993; Bronzino, 1999), we evaluate the relative power in each frequency band after normalizing by the total power. Thus, Kurth et al report an absolute increase in the delta power in the frontal regions over time; after correcting for amplitude variations, we find a relative decrease in delta power in both frontal and occipital regions between childhood and adolescence. Similarly, we find an increase in NREM theta power in the occipital region over adolescence, while others have reported decreased absolute theta power over this period of development (Campbell and Feinberg, 2009; Baker et al., 2011).

The function of cortical sleep rhythms has been studied extensively and remains an area of active research. Many of the rhythms present during sleep, including slow waves (delta), 
sleep spindles (beta/sigma) and gamma frequencies, are likely to support processes of learning and memory consolidation (Gais et al., 2002; Stickgold, 2005; Steriade, 2006; Fifer et al, 2010; Dragoi 2011; Uhlhaas and Singer, 2006; Roux et al., 2012). Some have proposed that the shifts in cortical rhythms observed over early development may reflect processes of synaptogenesis and pruning (Campbell and Feinberg, 2009; Tarokh et al., 2011; Kurth et al., 2010; Baker et al, 2011), early sensorimotor circuit formation (Khazipov et al., 2004), receptor modification (Uhlhaas and Singer, 2010) or represent the gradual emergence of self-awareness (Buzsaki, 2006). The emergence of discrete bands of oscillatory activity in early infancy and childhood may also reflect the maturation of distinct cortical generators producing the observed rhythms (Bollimunta et al., 2011). As each of these sleep rhythms emerge in an age-specific sequence with regional prominence, our data support the idea that specific rhythms may play key roles during critical periods of cognitive development and provides a physiological map through which to target key rhythms during development (Hensch, 2005; Espinosa and Stryker, 2012).

Increasingly, physiological coupling between brain regions is observed to enhance communication, establishing long-range networks that drive or support behavior (Fries, 2005; Hipp et al., 2011; Uhlhaas and Singer, 2006). Network measures allow characterization of the organization and features of the complex coupling patterns observed in EEG recordings. Here we find that broadband EEG functional networks increase in density over development, with an accelerated rise present after age 2 years. These broadband networks, which are dominated by low frequency delta and theta activity, are concomitantly sparser with a lower clustering coefficient with age. In contrast, high gamma frequency networks decreased in density with increased clustering with age. These observations fit with extensive work suggesting that low frequency cortical networks integrate longer-range neuronal assembles, while higher frequency networks are more spatially restricted (Buszaki and Watson, 2012). Physiologically, slower oscillations provide longer windows to integrate more neuronal assemblies over a larger cortical volume. The changes in network density in the low and high frequencies that we observed over development may reflect anatomical maturation processes, which could include white matter myelination and synaptic pruning which persist through adolescence (Hermoye et al., 2006, Huttenlocher and Dabholkar, 1997). In addition, recent work suggests that the maturation of extensive GABAergic interneuron assemblies likely plays a pivotal role in orchestrating the cortical field oscillations and network synchronization patterns observed here (Fritschy, 2008; Le Magueresse and Monyer, 2013).The selective maturation of these inhibitory circuits has been observed to occur in distinct steps over development, independent of environmental input (Ben-Ari et al., 2012; Baho and Cristo, 2012; Magueresse and Monyer, 2013).

In addition to the intrinsic biological events shaping cortical networks, some spontaneous cortical oscillations may require environmental input for appropriate maturation. Spindle bursts, triggered by muscle twitches in early development, are proposed to lay the circuitry for mature sensorimotor networks (Khazipov et al., 2004). Similarly, visual input is required for the maturation of cortical circuits in the visual cortex (Katz and Shatz, 1993). Our observation of stereotyped sequences of functional network topologies suggests that a predictable program directs the wiring of physiological networks over normal development. 
This emerging network may reflect stages of cellular, synaptic, and behavioral maturation, perhaps providing the dynamic scaffolding to support experience-expectant, adaptive cortical networks across each stage of neurological development.

Although spatially restricted patterns could be discerned in both the spectral and network analysis, anatomical correlation is limited by the low spatial resolution that results from spatial blurring of the voltage signal at the scalp and low density spatial sampling (Nunez, 2006). High density scalp EEG recordings and MEG recordings would allow better spatial resolution and approximation of rapid cortical dynamics for more accurate anatomical correlation. In addition, interpretation of high frequency activity in the spontaneous scalp EEG remains controversial. Many authors contend that gamma rhythms cannot be confidently identified due to the brain's inherent $1 /$ f properties and spatial filtering (Nunez and Srinivasan, 2010; McMenamin et al., 2011; Muthukumaraswamy et al., 2013), while others have reported success identifying focal gamma rhythms at the scalp surface (Ball et al,. 2008; Darvas et al., 2010; Andrade-Valenca et al., 2011). We observed marked increases in gamma power during childhood and adolescence, consistent with prior work in awake children (Uhlhaas et al., 2009). Although we applied conservative measures to remove subjects with possible muscle artifact, the results in the high frequencies should be interpreted with caution as muscle artifact cannot be definitively excluded. Secondary use of clinical data captured during neuromuscular blockade could help determine the extent that muscle artifact may contribute to these findings. Notably, all high gamma spectral and coherence measures were repeated excluding the studies sampled at $200 \mathrm{~Hz}$ ( $\mathrm{n}=52$ excluded) and qualitatively similar results were found. Furthermore, in our analysis, we evaluate only linear coupling between the recorded EEG activities. Study of nonlinear dynamics, including cross frequency coupling (Canolty and Knight, 2010), may contribute further to revealing and understanding the complex dynamics underlying brain organization and emergent properties of adaptive patterning and cortical network formation. In addition, although we employed strict inclusion and exclusion criteria to identify healthy subjects, because this study drew from a population of children referred for diagnostic evaluation, these subjects may not represent a community population sample. Finally, by undertaking a cross-sectional study, we were able to evaluate the maturation of cortical physiology at the population-level with short sampling intervals across an 18 year span. Although our findings are consistent with longitudinal work obtained across shorter intervals (Campbell and Feinberg, 2009; Tarokh et al., 2010; Sankupellay et al., 2011; Baker et al., 2012), longitudinal analysis would be required to confirm that the observed patterns hold within each individual.

In summary, we have characterized the rapidly developing cortical rhythms and functional brain networks from early infancy through adolescence in a large population of normal children during sleep. We found that cortical rhythms and functional networks change dramatically and predictably over infancy and childhood. These changes are evident within routine EEG recordings across a time span of months and years and provide a foundation upon which to better understand normal physiological brain development. Such cortical measures may also provide a sensitive clinical tool to interrogate and assess cortical health in the maturing brain. Future work is needed to tie these events to the sequence of adaptive behavioral and cognitive skills observed across development and to better understand how alterations in these precisely timed sequences may relate to and anticipate disease. 


\section{Acknowledgments}

This work was supported by the NIH K12 Neurological Sciences Academic Development Award (CJC), Harvard/MIT Health Sciences and Technology, Beth Israel Deaconess Medical Center Clinical Investigator Training Program in collaboration with Pfizer, Inc. and Merck $\mathrm{Co}(\mathrm{CJC})$, a Career Award at the Scientific Interface from the Burroughs Wellcome Fund (MAK), and a grant from the US NIH, NINDS NS062092 (SSC).

\section{References}

Anders TF. The infant sleep profile. Neuropadiatrie. 1974; 5:425-42. [PubMed: 4377797]

Andrade-Valenca LP, Dubeau F, Mari F, Zelmann R, Gotman J. Interictal scalp fast oscillations as a marker of the seizure onset zone. Neurology. 2011; 77:524-31. [PubMed: 21753167]

Baho E, Di Cristo G. Neural activity and neurotransmission regulate the maturation of the innervation field of cortical GABAergic interneurons in an age-dependent manner. J Neurosci. 2012; 32:911-8. [PubMed: 22262889]

Baker FC, Turlington SR, Colrain I. Developmental changes in the sleep electroencephalogram of adolescent boys and girls. J Sleep Res. 2012; 21:59-67. [PubMed: 21668552]

Ball T, Demandt E, Mutschler I, Neitzel E, Mehring C, Vogt K, et al. Movement related activity in the high gamma range of the human EEG. Neuroimage. 2008; 41:302-10. [PubMed: 18424182]

Baria AT, Baliki MN, Parrish T, Apkarian AV. 2011 Anatomical and functional assemblies of brain BOLD oscillations. J Neurosci. 2011; 31:7910-7919. [PubMed: 21613505]

Ben-Ari Y, Woodin MA, Sernagor E, Cancedda L, Vinay L, Rivera C, et al. Refuting the challenges of the developmental shift of polarity of GABA actions: GABA more exciting than ever! Front Cell Neurosci. 2012; 6:35. [PubMed: 22973192]

Benjamini Y, Hochberg Y. Controlling the false discovery rate: a practical and powerful approach to multiple testing. J Roy Statist Soc Ser B (Methodological). 1995; 57:289-300.

Bollimunta A, Mo J, Schroeder CE, Ding M. Neuronal mechanisms and attentional modulation of corticothalamic a oscillations. J Neurosci. 2011; 31:4935-43. [PubMed: 21451032]

Boersma M, Smit DJ, de Bie HM, Van Baal GC, Boomsma DI, de Geus EJ, et al. Network analysis of resting state EEG in the developing young brain: structure comes with maturation. Hum Brain Mapp. 2011; 32:413-25. [PubMed: 20589941]

Bronzino, JD. The biomedical engineering handbook. 2. CRC Press; Boca Raton, NO: 2000.

Buzsáki G. Large-scale recording of neuronal ensembles. Nat Neurosci. 2004; 7:446-51. [PubMed: 15114356]

Buszaki, G. Rhythms of the Brain. Oxford University Press; New York: 2006.

Buzsaki G, Draguhn A. Neuronal oscillations in cortical networks. Science. 2004; 304:1926-1929. [PubMed: 15218136]

Buzsaki G, Watson BO. Brain rhythms and neural syntax: implications for efficient coding of cognitive content and neuropsychiatric disease. Dialogues Clin Neurosci. 2012; 14:345-367. [PubMed: 23393413]

Campbell IG, Feinberg I. Longitudinal trajectories of non-rapid eye movement delta and theta EEG as indicators of adolescent brain maturation. PNAS. 2009; 106:5177-5180. [PubMed: 19307577]

Canolty RT, Knight RT. The functional role of cross-frequency coupling. Trends Cogn Sci. 2010; 11:506-15. [PubMed: 20932795]

Chu CJ, Kramer MA, Pathmanathan J, Bianchi MT, Westover MB, Wizon L, et al. Emergence of stable functional networks in long-term human electroencephalography. J Neurosci. 2012; 32:2703-13. [PubMed: 22357854]

Darvas F, Scherer R, Ojemann JG, Rao RP, Miller KJ, Sorensen LB. High gamma mapping using EEG. Neuroimage. 2010; 49:930-8. [PubMed: 19715762]

Dragoi G, Tonegawa S. Preplay of future place cell sequences by hippocampal cellular assemblies. Nature. 2011; 469:397-401. [PubMed: 21179088]

Eeg-Olofsson O. The development of the electroencephalogram in normal adolescents from the age of 16 through 21 years. Neuropadiatrie. 1971; 3:11-45. [PubMed: 5170965] 
Eeg-Olofsson O. Longitudinal developmental course of electrical activity of brain. Brain Dev. 1980; 2:33-44. [PubMed: 7416440]

Espinosa JS, Stryker MP. Development and plasticity of the primary visual cortex. Neuron. 2012; 75:230-49. [PubMed: 22841309]

Faust K. Very local structure in social networks. Sociol Methodol. 2007; 37:209-256.

Feinberg I, De Bie E, Davis NM, Campbell IG. Topographic differences in the adolescent maturation of the slow wave EEG during NREM sleep. Sleep. 2011; 34:325, 333. [PubMed: 21358849]

Fifer WP, Byrd DL, Kaku M, Eigsti IM, Isler JR, Grose-Fifer J, et al. Newborn infants learn during sleep. Proc Natl Acad Sci U S A. 2010; 107:10320-3. [PubMed: 20479232]

Freeman WJ, Rogers LJ, Holmes MD, Silbergeld DL. Spatial spectral analysis of human electrocorticograms including the alpha and gamma bands. J Neurosci Methods. 2000; 95:111-21. [PubMed: 10752481]

Fries P. A mechanism for cognitive dynamics: neuronal communication through neuronal coherence. Trends in Cog Sciences. 2005; 9:474-80.

Fritschy JM. Epilepsy, E/I Balance and GABA(A) Receptor Plasticity. Front Mol Neurosci. 2008; $1: 5$. [PubMed: 18946538]

Gais S, Mölle M, Helms K, Born J. Learning-dependent increases in sleep spindle density. J Neurosci. 2002; 22:6830-4. [PubMed: 12151563]

Gasser T, Schuller JC, Gasser US. Correction of muscle artefacts in the EEG power spectrum. Clin Neurophys. 2005; 116:2044-2050.

Gasser T, Verleger R, Bächer P, Sroka. Development of the EEG of school-age children and adolescents. I. Analysis of band power. Electroencephalogr Clin Neurophysiol. 1988; 69:91-9. [PubMed: 2446839]

Gaudreau H, Carrier J, Montplaisir J. Age-related modifications of NREM sleep EEG: from childhood to middle age. J Sleep Res. 2001; 10:165-172. [PubMed: 11696069]

Gray CM, König P, Engel AK, Singer W. Oscillatory responses in cat visual cortex exhibit intercolumnar synchronization which reflects global stimulus properties. Nature. 1989; 338:334. [PubMed: 2922061]

He BJ, Raichle ME. The fMRI signal, slow cortical potential and consciousness. Trends Cogn Sci. 2009; 13:302-309. [PubMed: 19535283]

He BJ, Zempel JM, Snyder AZ, Raichle ME. The temporal structures and functional significance of scale-free brain activity. Neuron. 2010; 66:353-69. [PubMed: 20471349]

Hensch TK. Critical period plasticity in local cortical circuits. Nat Rev Neurosci. 2005; 11:877-88. [PubMed: 16261181]

Hermoye L, Saint-Martin C, Cosnard G, Lee SK, Kim J, Nassogne MC, et al. Pediatric DTI: normal database and observation of the white matter maturation in early childhood. Neuroimage. 2006; 29:493-504. [PubMed: 16194615]

Hesdorffer DC, Logroscino G, Cascino G, Annegers JF, Hauser WA. Risk of unprovoked seizure after acute symptomatic seizure: effect of status epilepticus. Ann Neurol. 1988:908-912.

Hipp JF, Engel AK, Siegel M. Oscillatory synchronization in large-scale cortical networks predicts perception. Neuron. 2011; 69:387-96. [PubMed: 21262474]

Huttenlocher PR, Dabholkar AS. Regional differences in synaptogenesis in human cerebral cortex. J Comp Neurol. 1997; 387:167-178. [PubMed: 9336221]

Jenni OG, Carskadon MA. Spectral analysis of the sleep electroencephalogram during adolescence. Sleep. 2004; 27:774-83. [PubMed: 15283014]

Jenni OG, van Reen E, Carskadon MA. Regional differences of the sleep electroencephalogram in adolescents. J Sleep Res. 2005; 14:141-7. [PubMed: 15910511]

Katz LC, Shatz CJ. Synaptic activity and the construction of cortical circuits. Science. 1996; 274:1133-1138. [PubMed: 8895456]

Khazipov R, Sirota A, Leinekugel X, Holmes GL, Ben-Ari Y, Buzsaki G. Early motor activity drives spindle bursts in the developing somatosensory cortex. Nature. 2004; 432;9:758-761.

Komuro H, Rakic P. Modulation of neuronal migration by NMDA receptors. Science. 1993; 260:95-7. [PubMed: 8096653] 
Kramer MA, Eden UT, Cash SS, Kolaczyk ED. Network inference with confidence from multivariate time series. Phys Rev E Stat Nonlin Soft Matter Phys. 2009; 79:061916. [PubMed: 19658533]

Kramer MA, Cash SS. Epilepsy as a disorder of cortical network organization. Neuroscientist. 2012; 18:360-72. [PubMed: 22235060]

Kriegstein AR. Constructing circuits: neurogenesis and migration in the developing neocortex. Epilepsia. 2005; 46:15-21. [PubMed: 16201991]

Kuks JB, Vos JE, O'Brien MJ. EEG coherence functions for normal newborns in relation to their sleep state. Electroencephalogr Clin Neurophysiol. 1988; 69:295-302. [PubMed: 2450728]

Kurth S, Ringli M, Geiger A, LeBourgeois M, Jenni OG, Huber R. Mapping of cortical activity in the first two decades of life: a high-density sleep electroencephalogram study. J Neurosci. 2010; 30:13211-13219. [PubMed: 20926647]

Law SK. Thickness and resistivity variations over the upper surface of the human skull. Brain Topogr. 1993; 6:99-109. [PubMed: 8123431]

Le Magueresse C, Monyer H. GABAergic Interneurons Shape the Functional Maturation of the Cortex. Neuron. 2013; 77:388-405. [PubMed: 23395369]

Micheloyannis S, Vourkas M, Tsirka V, Karakonstantaki E, Kanatsouli K, Stam CJ. The influence of ageing on complex brain networks: a graph theoretical analysis. Hum Brain Mapp. 2009; 30:2008. [PubMed: 17990300]

McMenamin BW, Chackman AJ, Gerischar LL, Davidson RJ. Electromyogenic artifacts and electroencephalographic inferences revisited. Neuroimage. 2011; 54:4-9. [PubMed: 20981275]

Muthukumaraswamy SD. High-frequency brain activity and muscle artifacts in MEG/EEG: a review and recommendations. Front Hum Neurosci. 2013; 7:1-11. [PubMed: 23355817]

Myers MM, Grieve PG, Israelit A, Fifer WP, Isler JR, Darnall RA, et al. Developmental profiles of infant EEG: overlap with transient cortical circuits. Clin Neurophys. 2012; 123:1502-11.

Newman, M. Networks: an introduction. 1. USA: Oxford University Press; 2010.

Piantoni G, Astill RG, Raymann RJEM, Vis JC, Coppens JE, Someren EJWV. Modulation of gamma and spindle-range power by slow oscillations in scalp sleep EEG of children. Int J Psychophys. 2013; 89:252-8.

Nunez, PL.; Srinivasan, R. Electric fields of the brain: the neurophysics of EEG. Oxford University Press; Oxford: 2006.

Nunez PL, Srinivasan R. Scale and frequency chauvinism in brain dynamics: too much emphasis on gamma band oscillations. Brain Structu and Funct. 2010; 215:67-71.

O'Keefe J, Recce ML. Phase relationship between hippocampal place units and the EEG theta rhythm. Hippocampus. 1993; 3:317-30. [PubMed: 8353611]

Partha, M.; Hemant, B. Observed Brain Dynamics. Oxford University Press; New York: 2008.

Rosenberg RS, Van Hout S. The American Academy of Sleep Medicine inter-scorer reliability program: sleep stage scoring. J Clin Sleep Med. 2013; 9:81-7. [PubMed: 23319910]

Roux F, Wibral M, Mohr HM, Singer W, Uhlhaas PJ. Gamma-band activity in human prefrontal cortex codes for the number of relevant items maintained in working memory. J Neurosci. 2012; 32:12411-20. [PubMed: 22956832]

Rubinov M, Sporns O. Complex network measures of brain connectivity: uses and interpretations. Neuroimage. 2010; 52:1059-69. [PubMed: 19819337]

Sankupellay M, Wilson S, Heussler HS, Parsley C, Yuill M, Dakin C. Characteristics of sleep EEG power spectra in healthy infants in the first two years of life. Clin Neurophys. 2011; 122:236-243.

Scher MS. Ontogeny of EEG-sleep from neonatal through infancy periods. Sleep Med. 2008; 9:61536. [PubMed: 18024172]

Scher MS, Loparo KA. Neonatal EEG/sleep state analyses: a complex phenotype of developmental neural plasticity. Dev Neurosci. 2009; 31:259-75. [PubMed: 19546563]

Shinnar S, Glauser T. Febrile seizures. J Child Neuro. 2002; 17:S44-S52.

Silber MH, Ancoli-Israel S, Bonnet MH, Chokroverty S, Grigg-Damberger MM, Hirshkowitz M, et al. The visual scoring of sleep in adults. J Clin Sleep Med. 2007; 3:121-31. [PubMed: 17557422]

Singer W. Neuronal synchrony: a versatile code for the definition of relations? Neuron. 1999; 24:49;65, 111-125. [PubMed: 10677026] 
Steriade M. Grouping of brain rhythms in corticothalamic systems. Neuroscience. 2006; 137:1087106. [PubMed: 16343791]

Smit DJ, Boersma M, Schnack HG, Micheloyannis S, Boomsma DI, Hulshoff Pol HE, et al. The brain matures with stronger functional connectivity and decreased randomness of its network. PLoS One. 2012; 7:e36896. [PubMed: 22615837]

Sterman MB, Harper RM, Havens B, Hoppenbrouwers T, McGinty DJ, Hodgman JE. Quantitative analysis of infant EEG development during quiet sleep. Electroencephalogr Clin Neurophysiol. 1977; 43:371-85. [PubMed: 70338]

Stickgold R. Sleep-dependent memory consolidation. Nature. 2005; 437:1272-8. [PubMed: 16251952]

Sun L, Grützner C, Bölte S, Wibral M, Tozman T, Schlitt S, et al. Impaired gamma-band activity during perceptual organization in adults with autism spectrum disorders: evidence for dysfunctional network activity in frontal-posterior cortices. J Neurosci. 2012; 11;32:9563-73.

Tarokh L, Carskadon MA, Achermann P. Developmental changes in brain connectivity assessed using the sleep EEG. Neuroscience. 2010; 171:622-34. [PubMed: 20833232]

Tarokh L, Van Reen E, LeBourgeois M, Seifer R, Carskadon MA. Sleep EEG provides evidence that cortical changes persist into late adolescence. Sleep. 2011; 34:1385-93. [PubMed: 21966070]

Thatcher RW, North DM, Biver CJ. Development of cortical connections as measured by EEG coherence and phase delays. Hum Brain Mapp. 2008; 29:1400-15. [PubMed: 17957703]

Uhlhaas PJ, Singer W. Neural synchrony in brain disorders: relevance for cognitive dysfunctions and pathophysiology. Neuron. 2006; 52:155-68. [PubMed: 17015233]

Uhlhaas PJ, Roux F, Singer W, Haenschel C, Sireteanu R, Rodriguez E. The development of neural synchrony reflects late maturation and restricting of functional networks. PNAS. 2009; 106:98669871. [PubMed: 19478071]

Uhlhaas PJ, Singer W. Abnormal neural oscillations and synchrony in schizophrenia. Nat Rev Neurosci. 2010; 11:100-13. [PubMed: 20087360] 


\section{HIGHLIGHTS}

- The sleep EEG provides an in vivo assay of spontaneous cortical activity across post-natal development.

- Early brain development is marked by dramatic alterations in discrete cortical rhythms.

- Stereotyped integration patterns between brain rhythms emerge across early brain development. 

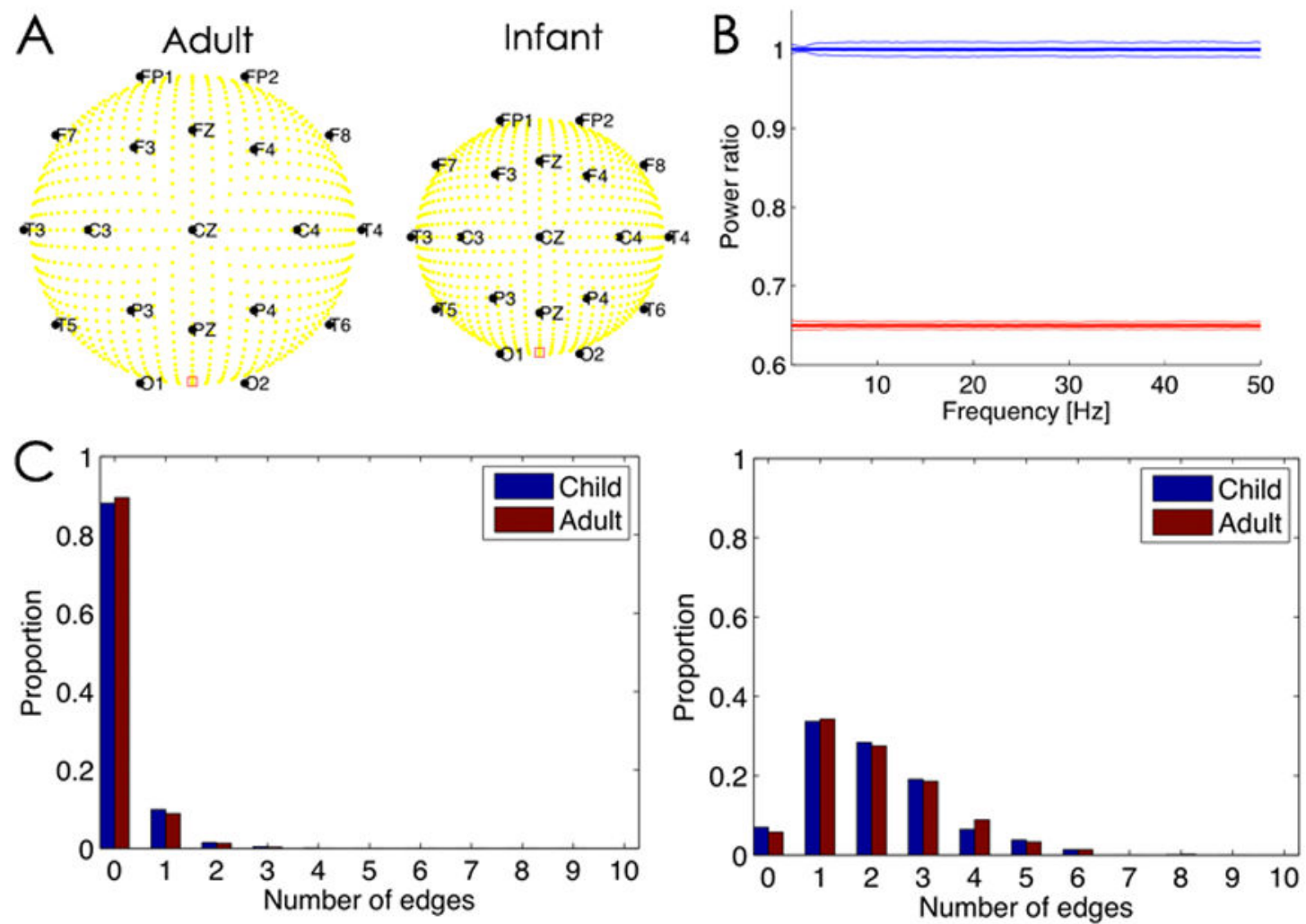

Figure 1. Normalization of power to mitigate impact of skull geometry over development A. Illustration of the head geometries in the two model configurations, Adult (left) and Infant (right). The yellow circles denote dipole sources in the cortex, and the black circles scalp electrode locations (with labels). The red square between $\mathrm{O} 1$ and $\mathrm{O} 2$ denotes the physical reference. B. The ratio of the power spectrum computed in the Adult model configuration divided by the Infant model configuration. The average power is computed for a $2 \mathrm{~s}$ interval over all electrodes in each configuration, and then the ratio is determined. The blue line is the power ratio for the normalized spectra. The thick line indicates the mean ratio and the thin lines the $95 \%$ confidence intervals over 1000 instantiations of $2 \mathrm{~s}$ of pink noise dipole source activity. The sampling frequency is $512 \mathrm{~Hz}$. The mean ratio is near 1 , which suggests that the normalization prevents alterations in power due to changes in head geometry. The red line is the power ratio of spectra that have not been normalized; here the mean is smaller because there is less power in the Adult spectra due to the spatial blurring of the thicker skull. C. The number of edges detected in the inferred functional networks depends on the dipole source activity, regardless of head geometry. (Left) When the dipole sources consist of uncorrelated pink noise, both head geometries (Adult and Child, see legend) tend to detect one or fewer edges. (Right) When a subset of dipole sources possess correlated activity, both head geometries detect one or more edges. 

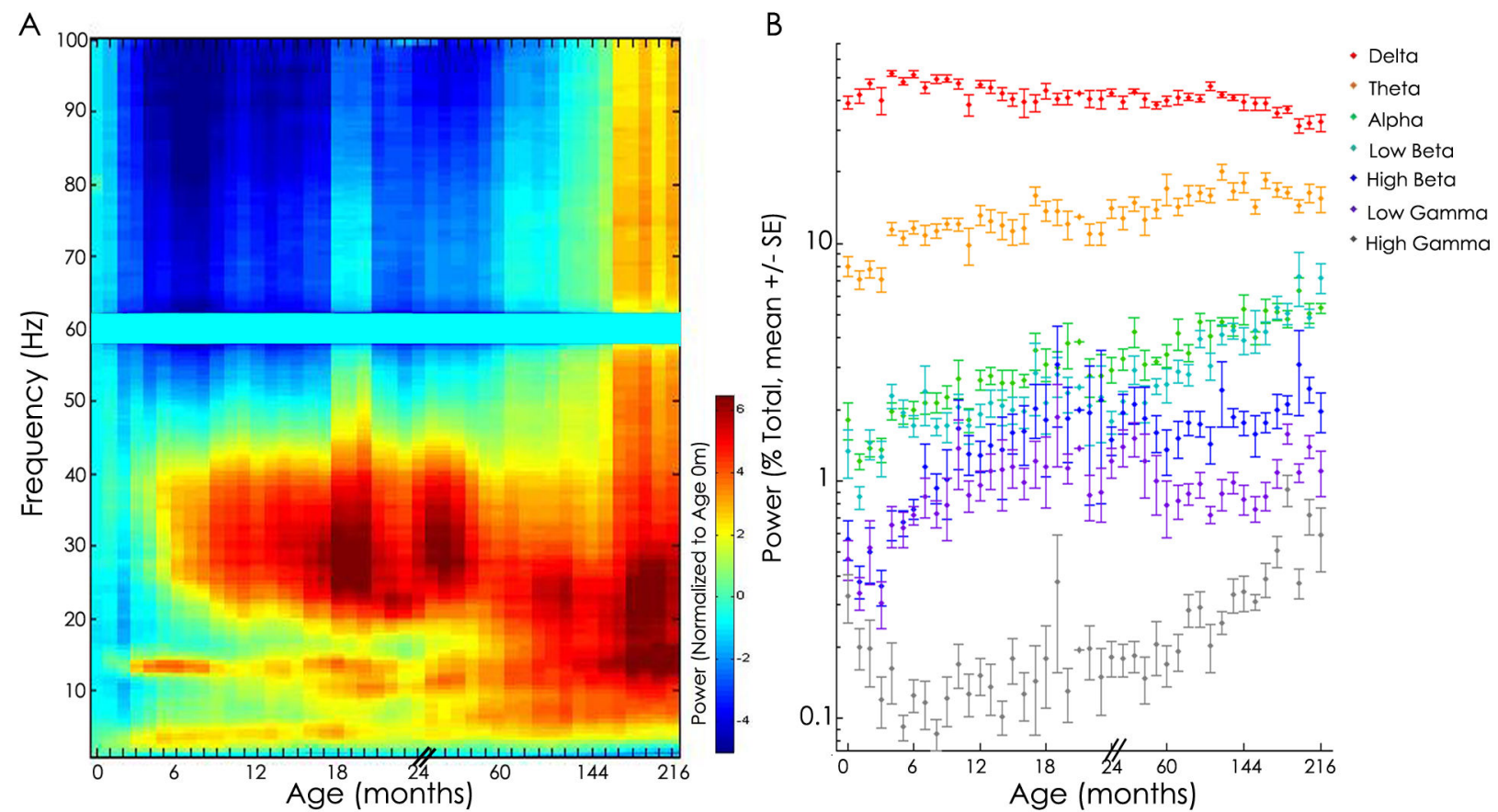

Figure 2. Cortical power spectra across development

A. Visualization of power spectra. Relative power in decibels (averaged across all electrodes) is plotted as a function of age and frequency, averaged across adjacent age bins, and normalized to age 0 months. In general, lower frequencies $(<5 \mathrm{~Hz})$ dominate over early infancy and higher frequencies increase in power over childhood. Additional structure is also present. For example, a prominent increase in power is present in the low beta band during infancy and in the high beta and low gamma bands during the second year of life. $\mathbf{B}$. Average relative power in five frequency bands. The percent total power (+/- SEM) across seven classical frequency bands is plotted for each age group (note, the x-axis is not continuous; please see Methods for age bins). Complex dynamics are observed, but in general, delta power is highest in infancy and power in the higher frequency bands $(>4 \mathrm{~Hz})$ significantly increases with age (ANOVA, $\mathrm{p}<0.0001$ for each frequency band). Note: Separate color schemes reflect power in 1A and frequency band in $1 \mathrm{~B}$. 
A

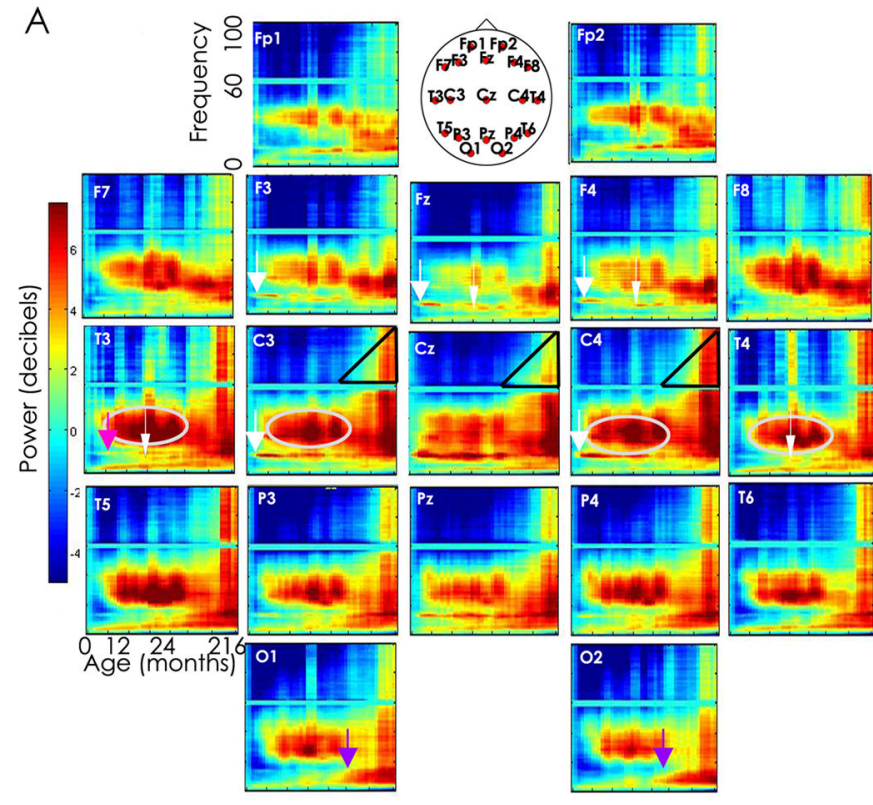

B

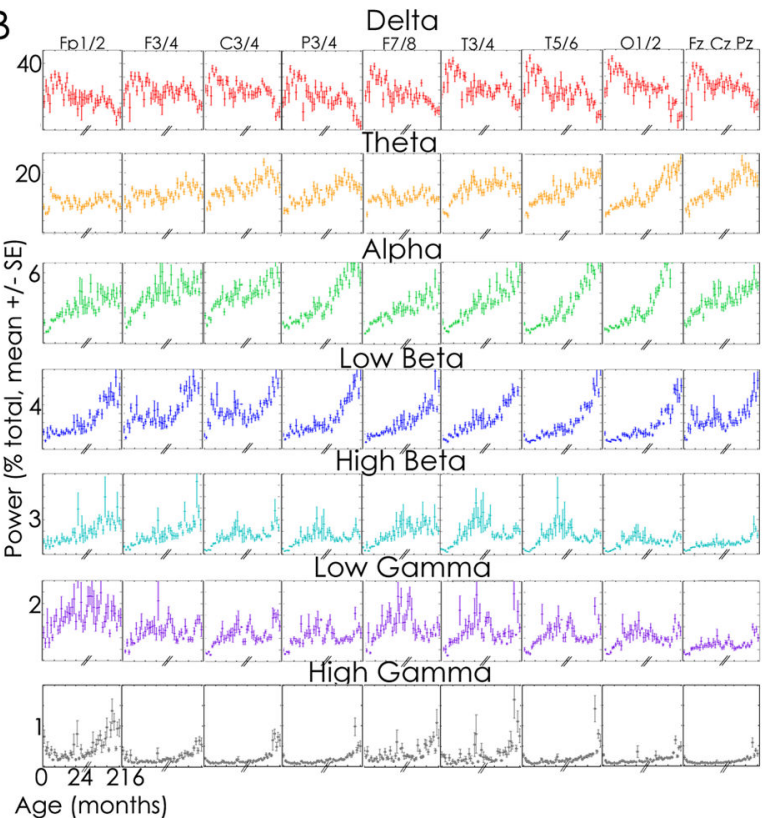

Figure 3. A. Regional power spectra

Average relative power in decibels is plotted as a function of age and frequency for each voltage tracing, averaged across adjacent age bins and normalized to age 0 months. We plot the average electrode location on the head schematic for representative location of the electrode sensors. Several regionally specific frequency dynamics are observed. For example, prominent beta and gamma activity is present in central and temporal regions from $6 \mathrm{~m}$ to $5 \mathrm{y}$ (silver circles), while prominent alpha and theta activity appears at $5 \mathrm{y}$ in the posterior regions (purple arrows). Two distinct bands of activity are present in the midline and frontocentral region at age 2 months (14 Hz, broad white arrows) and 12-18 months (11 $\mathrm{Hz}$, narrow white arrows) while 3 distinct bands (centered at $5 \mathrm{~Hz}, 11 \mathrm{~Hz}$, and $14 \mathrm{~Hz}$ ) are present in the left temporal region at age 18 months (pink arrow). High frequency power $(>60 \mathrm{~Hz})$ increases steadily after age 5 years, most prominently in the central regions (black triangles). B. Regional power spectra in classical frequency bands. Average power is plotted for each age in each frequency band for each electrode derivation. Results of homologous electrodes are averaged for ease of visualization. Marked spatial structure is present in the relative power in each frequency over development. In the delta band, the dramatic rise and fall in power in the first 15 months followed by a second fall in adolescents is evident in all electrode locations, though most prominent in the temporal and occipital electrodes. In the theta band, prominent increases in theta activity are observed through childhood in the central and parietal regions, which persist through adolescence in the occipital regions. In the alpha band, early increases in relative power are present in infancy, and most prominent in the frontocentral regions, followed by a dramatic increase in power in the parietal, temporal and occipital regions over childhood and adolescence. In higher frequencies, a peak in low beta activity is present in the frontocentral regions during infancy, a prominent peak in high beta activity is present in the temporal regions during the second year of life, and a rise in low gamma activity is present in the frontal region through 2 years before a subsequent relative decline. 


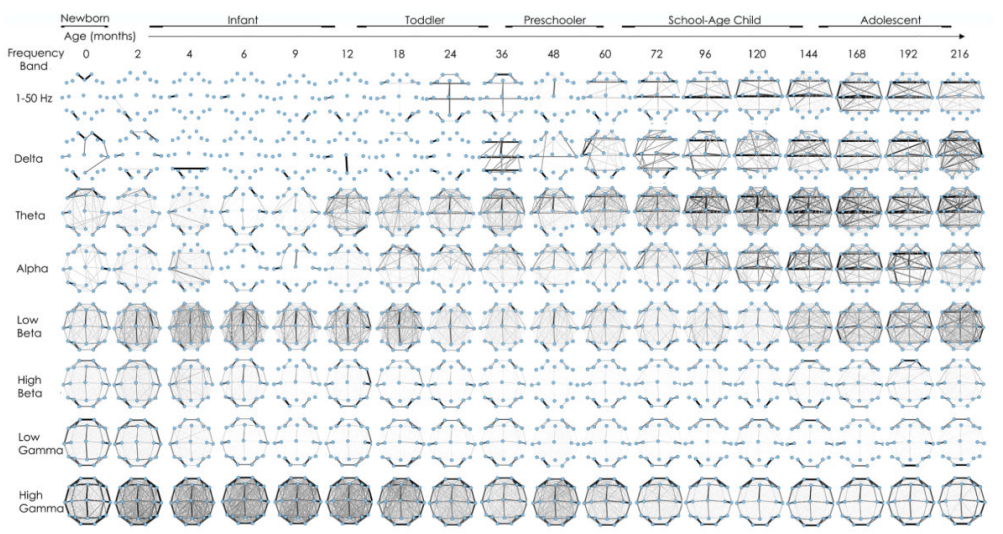

Figure 4. Functional network topology across development during sleep

The average networks are plotted for age groups ranging from 0 months through 18 years (averaged across adjacent age bins) using cross correlation (broadband, 1-50 Hz) and coherence measures for narrowband frequencies (delta, theta, alpha, beta, high beta, low gamma, high gamma) frequencies. Nodes represent average location of voltage recordings used in the nearest neighbor Laplacian reference. Edges represent presence of significant coupling between cortical voltage recordings. The width of the edge is drawn proportional to its weight, such that the most persistent edges present over the recording epoch are dark and thick. Striking topological organization is present across development in each frequency band (please see text). 


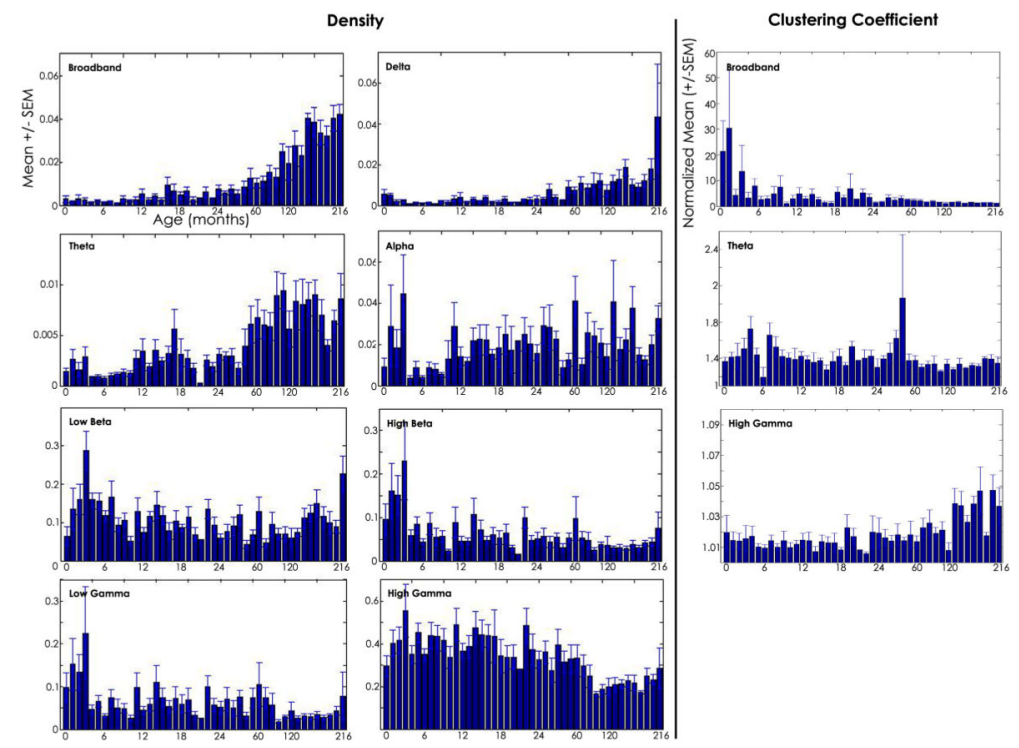

Figure 5. Functional network characteristics across development

Left: The mean density (+/- standard error of the mean, $y$-axis) is plotted for each age group (months, $\mathrm{x}$-axis) for the broadband and narrow band networks. The broadband networks represent a summary statistic of connectivity strength across all frequencies. Here we see that in general, network density increases with age, rapidly increasing after age 60 months. When narrower frequency bands are evaluated, more structure is present. Right: The mean global clustering coefficient (normalized to 500 random networks with equal density, +/standard error of the mean) is plotted for each age group for three frequency bands in which some structure across development is present. 


\section{Table 1}

Indications for EEG

\begin{tabular}{|c|c|}
\hline Diagnosis & $\mathbf{N}$ \\
\hline Provoked Seizure & 88 \\
\hline Syncope & 40 \\
\hline Headaches & 35 \\
\hline Breathholding spells & 21 \\
\hline Non-epileptic staring & 20 \\
\hline Gastrointestinal reflux & 18 \\
\hline Behavioral events & 14 \\
\hline Stereotypies/tics & 14 \\
\hline Shuddering spells & 14 \\
\hline Transient unresponsiveness & 13 \\
\hline Sleep myoclonus & 13 \\
\hline Nonspecific movements & 12 \\
\hline Unusual eye movements & 9 \\
\hline Uncertain indication & 8 \\
\hline Sleep phenomenon & 6 \\
\hline Acute life-threatening event & 5 \\
\hline Tremors & 5 \\
\hline Vertigo & 5 \\
\hline Dizziness & 4 \\
\hline Transient stiffening & 4 \\
\hline Voluntary movements & 4 \\
\hline Altered mental status & 3 \\
\hline Apnea & 3 \\
\hline Falls & 3 \\
\hline Head nods & 3 \\
\hline Vomiting & 3 \\
\hline Hypoglycemia & 2 \\
\hline Startles & 2 \\
\hline Visual phenomenon & 2 \\
\hline Aplastic anemia & 2 \\
\hline Bell's palsy & 1 \\
\hline Hypertonia & 1 \\
\hline Ingestion & 1 \\
\hline Intussusception & 1 \\
\hline Lyme disease & 1 \\
\hline Panic attack & 1 \\
\hline Polydipsia & 1 \\
\hline Suspected abuse & 1 \\
\hline Trauma & 1 \\
\hline
\end{tabular}

